\title{
Real-world comparative effectiveness of second-line ipilimumab for metastatic melanoma: a population-based cohort study in Ontario, Canada
}

Wei Fang Dai ${ }^{1,2}$, Jaclyn M. Beca ${ }^{1,2}$, Ruth Croxford ${ }^{3}$, Wanrudee Isaranawatchai ${ }^{2,4,5}$, Ines B. Menjak ${ }^{6,7}$, Teresa M. Petrella ${ }^{7}$, Nicole Mittmann', Craig C. Earle ${ }^{3}$, Scott Gavura', Timothy P. Hanna ${ }^{8,9,10+}$ and Kelvin K.W. Chan ${ }^{1,2,7^{*+}}$

\begin{abstract}
Background: For novel cancer treatments, effectiveness in clinical practice is not always aligned with clinical efficacy results. As such it is important to understand a treatment's real-world effectiveness. We examined real-world population-based comparative effectiveness of second-line ipilimumab versus non-ipilimumab treatments (chemotherapy or targeted treatments).

Methods: We used a cohort of melanoma patients receiving systemic treatment for advanced disease since April 2005 from Ontario, Canada. Patients were identified from provincial drug databases and the Ontario Cancer Registry who received second-line ipilimumab from 2012 to 2015 (treated) or second-line non-ipilimumab treatment prior to 2012 (historical controls). Historical controls were chosen, to permit the most direct comparison to pivotal trial findings. The cohort was linked to administrative databases to identify baseline characteristics and outcomes. Kaplan-Meier curves and multivariable Cox regression models were used to assess overall survival (OS). Observed potential confounders were adjusted for using inverse probability of treatment weighting (IPTW).

Results: We identified 329 patients with metastatic melanoma (MM) who had received second-line treatments (189 treated; 140 controls). Patients receiving second-line ipilimumab were older (61.7 years vs 55.2 years) compared to historical controls. Median OS were 6.9 (95\% Cl: 5.4-8.3) and 4.95 (4.3-6.0) months for ipilimumab and controls, respectively. The crude 1-year, 2-year, and 3-year OS probabilities were 34.3\% (27-41\%), 20.6\% (15-27\%), and 15.2\% (9.6-21\%) for ipilimumab and $17.1 \%(11-23 \%), 7.1 \%(2.9-11 \%)$, and $4.7 \%(1.2-8.2 \%)$ for controls. Ipilimumab was associated with improved OS (IPTW HR $=0.62 ; 95 \%$ Cl: $0.49-0.78 ; p<0.0001$ ).
\end{abstract}

Conclusions: This real-world analysis suggests second-line ipilimumab is associated with an improvement in OS for MM patients in routine practice.

Keywords: Real-world, Comparative effectiveness, Metastatic melanoma, Immunotherapy

\footnotetext{
* Correspondence: kelvin.chan@sunnybrook.ca

tTimothy P. Hanna and Kelvin K.W. Chan contributed equally as senior authors.

${ }^{1}$ Cancer Care Ontario, Toronto, ON, Canada

${ }^{2}$ Canadian Centre for Applied Research in Cancer Control, Toronto, ON,

Canada

Full list of author information is available at the end of the article
}

(c) The Author(s). 2020 Open Access This article is licensed under a Creative Commons Attribution 4.0 International License, which permits use, sharing, adaptation, distribution and reproduction in any medium or format, as long as you give appropriate credit to the original author(s) and the source, provide a link to the Creative Commons licence, and indicate if changes were made. The images or other third party material in this article are included in the article's Creative Commons licence, unless indicated otherwise in a credit line to the material. If material is not included in the article's Creative Commons licence and your intended use is not permitted by statutory regulation or exceeds the permitted use, you will need to obtain permission directly from the copyright holder. To view a copy of this licence, visit http://creativecommons.org/licenses/by/4.0/. The Creative Commons Public Domain Dedication waiver (http://creativecommons.org/publicdomain/zero/1.0/) applies to the data made available in this article, unless otherwise stated in a credit line to the data. 


\section{Background}

The treatment effects of novel cancer therapies adopted into clinical practice (effectiveness) do not always align with clinical trial outcomes (efficacy) [1]. In particular, for many new cancer drugs, differences between efficacy and effectiveness are often not known, leading to uncertainty in clinical decision-making and drug funding decisions. As such, it is important to investigate the effectiveness of novel systemic therapies in routine practice, using population-based administrative databases, which are reflective of the real-world patient experience.

Ipilimumab, an anti-cytotoxic T-cell lympohocyte-4 monoclonal antibody, was the first immune checkpoint inhibitor therapy to show significant survival benefits in patients with unresectable or metastatic melanoma (MM) [2]. Prior to the introduction of ipilimumab, treatment options for MM patients typically include standard cytotoxic chemotherapy such as dacarbazine or temolozomide. Often, these treatments conferred limited benefit with phase II trial results showing median overall survival around 6.2 months and 1 year survival rate of 25.5\% [3]. In Canada, ipilimumab in the second-line setting was the first immunotherapy to receive regulatory approval and was publicly funded in most provinces since 2012. Funding for ipilimumab in Canada was subsequently extended to first-line use in 2015 and to combination therapy with nivolumab in 2019 .

In the pivotal trial for ipilimumab, Hodi et al. observed survival benefits (hazard ratio $(\mathrm{HR})=0.66$; $95 \%$ CI: 0.51-0.87) for patients receiving ipilimumab (median overall survival $(\mathrm{OS})=10.1$ months) compared to those receiving glycoprotein 100 peptide vaccine alone (median OS 6.4 months) [2]. Patients receiving ipilimumab had higher survival rates $(12$ months $=45.6 \%$; 18 months $=33.2 \%$; 24 months $=23.5 \%)$ than those receiving glycoprotein 100 alone $(12$ months $=25.3 \% ; 18$ months $=16.3 \% ; \quad 24$ months $=13.7 \%)$. Moreover, the ipilimumab group was observed to maintain longterm benefit, with a $20 \%$ survival rate up to the end of the follow up of 54 months. In an analysis of patients with at least two years of potential, $25 \%$ of patients in the ipilimumab group survived more than 2 years and 3 years since initial randomization [4]. In the control group 17 and $10 \%$ survived more than 2 and 3 years, respectively [4].

To date, only one published study has examined the real-world comparative effectiveness of second-line ipilimumab compared to standard chemotherapy. This study, conducted in Poland, found similar survival benefit as the trial (adjusted HR $=0.65$ ), but observed differences in median OS from the clinical trial experience, including attenuated median OS and less evident survival tail plateau compared to that observed in the trial $[2,5]$. Additionally, correction for possible lead-time bias or differences in patient comorbidity between groups was not undertaken and notably the comparison group was contemporary rather than historic. The issue of lead-time bias is especially relevant given that clinical enthusiasm for a new and effective first-in-class drug like ipilimumab could lead to earlier initiation of second line immunotherapy treatment compared to second-line chemotherapy, which could influence observed differences in survival between comparison groups. Moreover, given possible differences in case mix among jurisdictions, it is important to evaluate the real-world comparative effectiveness of second-ipilimumab in a North American population.

Using population-based data from the Canadian province of Ontario, we set out to quantify the real-world effectiveness of second-line ipilimumab in routine practice, as compared to efficacy observed in pivotal trials.

\section{Methods}

We conducted a population-based comparative retrospective cohort study of individuals treated for MM with second-line ipilimumab, chemotherapy, or targeted therapy between 2012 and 2015 in Ontario. Ontario is the largest province in Canada with a population of approximately 14 million people [6]. For patients with MM, the primary treatment is systemic treatments administered by cancer clinics and funded by the provincial government public drug programs.

\section{Study population}

Patients diagnosed with melanoma (International Classification of Disease for Oncology, 3rd edition - topography:C44) who started first-line systemic treatment for advanced melanoma on or after April 1, 2005 were identified from the Activity Level Reporting (ALR) systemic treatment database maintained by the provincial cancer agency, Cancer Care Ontario. The ALR database consists of population-wide systemic treatment records for cancer patients in Ontario. Patients who received first-line treatment with palliative intent were considered for inclusion, defined as the first non-interferon systemic therapy received by the patient. Since a treatment regimen might consist of multiple drugs provided on different days, a line of treatment was defined as consisting of all chemotherapy drugs given to a patient within four days of the start of treatment. The line of treatment was deemed to continue as long as the patient continued to receive at least one of the drugs. The data sources used to identify treatment regimens are presented in Additional file 1.

Patients were included in the study if they started a second line of treatment prior to September 13, 2012 (historical controls) or if they started a second line of 
treatment with ipilimumab between September 13, 2012 and March 31, 2015 (treated). Historical controls were chosen, to permit the most direct comparison to pivotal trial findings. Patients were excluded if they were younger than 18 at the time of their melanoma diagnosis, had been diagnosed with another prior cancer, received ipilimumab as part of their first-line treatment or received experimental treatment/clinical trial agents as part of their 2nd line of treatment. Potential treated patients were excluded if they received ipilimumab in combination with another treatment (Fig. 1). The index date was the date of the start of second-line treatment.

\section{Outcomes and covariates}

The study cohort was linked to administrative databases to determine baseline demographics and date of death. The primary outcome was overall survival, defined as the time from the start of second-line treatment until death. Patients were censored if they emigrated from Ontario or remained alive at the end of follow up on March 31, 2017. Patient demographic characteristics including age, sex, postal code, and date of death were obtained from the Registered Persons Database. Patient comorbidity was calculated using a weighted average of the Adjusted Clinical Groups (Johns Hopkins ACG(R) System, Version 10) which were based on diagnoses obtained from hospital discharge records and physician claims records in the 2 years prior to the index date, excluding cancer diagnoses [7]. In addition, the Charlson score was calculated based on diagnoses found in hospital discharge records in the 2 previous years, excluding cancer diagnoses [8]. Statistics Canada's 2016 census data was used to obtain rural/urban status of the patient's residence and neighborhood income quintile based on postal codes. Additional information on other systemic therapy and radiotherapy was obtained from the ALR and hospital discharge records. These datasets were linked using unique, encoded identifiers and analyzed at ICES.

\section{Statistical analysis}

For patient and treatment characteristics, mean and standard deviation or median and interquartile range were reported for continuous variables, and frequencies and percentages were calculated for categorical variables. Baseline characteristics of the ipilimumab-treated and control patients were compared using chi-square tests for binary covariates, Kruskal-Wallis tests for categorical variables, t-tests for normally distributed continuous variables, and Wilcoxon rank sum tests for skewed numerical variables.

To adjust for differences between groups, propensity scores were calculated using logistic regression, and were used to calculate inverse probability of treatment weights (IPTW) [9]. Baseline covariates used to calculate the final propensity scores were patient age, place of residence, the comorbidity score and Charlson score, prior radiation to the brain, prior radiation to other body

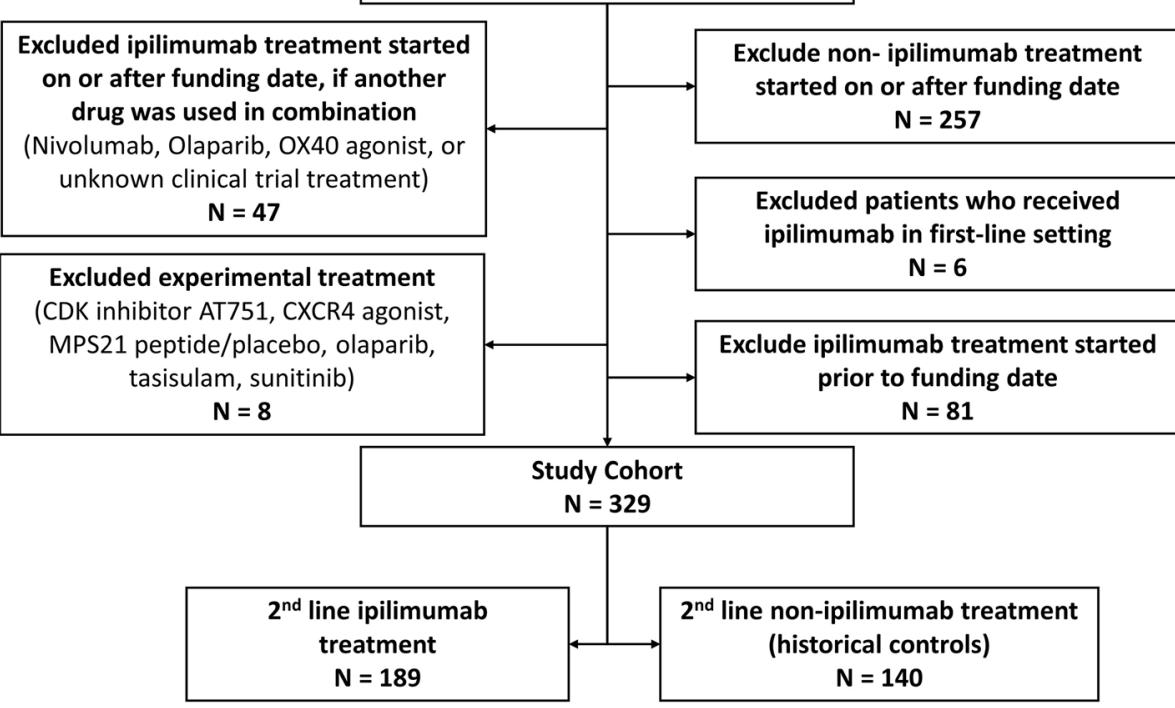

Fig. 1 Diagram of study cohort creation 
parts, and time from diagnosis to the start of second line treatment. Standardized differences for the IPTWweighted covariates were calculated. Standardized differences $\leq 0.10$ are generally considered to represent acceptable balance [9].

Overall survival was assessed using Kaplan-Meier method and log-rank test was performed to assess the difference between groups. Time to death between groups was also compared using a Cox proportional hazards model, weighting patients in each group by their IPTWs. Due to the use of IPTWs, the resulting hazard ratios estimate the average treatment effect, an estimate of the treatment effect if ipilimumab rather than the historical treatments were used as second line treatment in the population of melanoma patients who are similar to those included in this study. Sensitivity analyses were performed, incorporating third-line treatment (if any) into the survival analyses by i) adjusting for the use of third-line treatment as a time-varying confounder; ii) adjusting third-line checkpoint inhibitors treatment as a time-varying confounder; iii) censoring patients at the time of initiating third-line treatment; and iv) excluding patients who received third-line treatment.

Two-sided $p$-values $<0.05$ were considered to be statistically significant. SAS version 9.4 (SAS institute, Cary, North Carolina) was used for the analyses.

\section{Results}

\section{Study population}

A total of 728 patients received a second-line treatment for MM between September 13th, 2008 and March 31st, 2015. Of these identified patients, 329 patients met eligibility criteria and were included (Fig. 1). The final cohorts consist of 140 (42.6\%) control patients who received second-line treatment with chemotherapy or targeted therapy prior to the ipilimumab funding date and $189(57.4 \%)$ treated patients who received secondline ipilimumab treatment after the funding date.

\section{Baseline characteristics}

Baseline demographic and clinical characteristics are presented in Table 1 . In the total unweighted cohort, the mean age is 58.9 (SD: 16.4) years, $67.8 \%$ were males, and $87.5 \%$ lived in urban regions. At baseline, patients who are treated with second-line ipilimumab were older (61.7 years vs 55.2 years) compared to historical controls. There were no significant differences in sex, income quintile, comorbidities, and prior receipt of radiation between the ipilimumab-treated and the historical control patients.

The median time from diagnosis to initiating second-line treatment was 18 months (95\% CI: 8.438.5 months) for second-line ipilimumab patients and
32.5 months (95\% CI: $11.9-57.5$ months) for historical controls (Table 1). The median time between the end of first-line and the start of second-line treatment was shorter for patients receiving second-line ipilimumab (1 month vs 1.7 months; $p$-value <0.001) (Table 3). The majority of the historical controls received first-line chemotherapy $(78.6 \%)$, while a minority received first-line BRAF/MEK (9.3\%), with the remainder $(10 \%)$ receiving either non-ipilimumab immunotherapy or other treatments for first-line therapy. The majority of patients who received secondline ipilimumab (treated) received chemotherapy (63\%) or BRAF/MEK inhibitors $(32.3 \%)$ as their firstline treatment.

Weighted standardized difference between the treated and historical controls for all baseline characteristics were calculated after IPTW adjustment. All standardized differences were less than 0.1 with the exception of age, income quintile (medium and medium to high), and time from end of first-line treatment to start of secondline treatment.

\section{Treatment patterns}

Approximately half (49.2\%) of the patients receiving second-line ipilimumab completed all four planned doses of ipilimumab; the remaining $14.8 \%$ had one dose, $19.6 \%$ had 2 doses, and $13.2 \%$ had 3 doses. Amongst the historical controls, 127 patients received chemotherapy (e.g. dacarbazine and temozolomide) and other treatment (e.g. tyrosine kinase inhibitors), while 13 patients received BRAF/MEK (e.g. vemurafenib, dabrafenib). Amongst the study cohort of patients receiving secondline treatments, 38 (27.1\%) historical controls and 64 (35.5\%) ipilimumab patients proceeded to receive at least one third-line treatment. Of those patients who received third-line treatments, 27 (71\%) historical controls and 51 (76.1\%) ipilimumab patients received immunotherapy, while the remaining patients received chemotherapy or other treatments. Amongst the historical controls, the third-line immunotherapy received were mainly ipilimumab whereas the immunotherapy received by the cases were either nivolumab or pembrolizumab.

\section{Overall survival}

The cohort of patients were followed up until March 31st, 2017 with a median follow-up of 30.4 months (95\% CI: $27.9-37.7$ months) in second-line ipilimumab patients and 71.2 months (95\% CI: 70.3-116.5 months) in historical controls (Table 2). Crude median OS was 6.9 months (95\% CI: 5.4-8.3 months) and 4.9 months (95\% CI: 4.3-6.0 months) for patients receiving second-line ipilimumab and historical controls, respectively (Fig. 2a). The adjusted median OS is also greater in second-line ipilimumab (7.2 months; $95 \%$ 


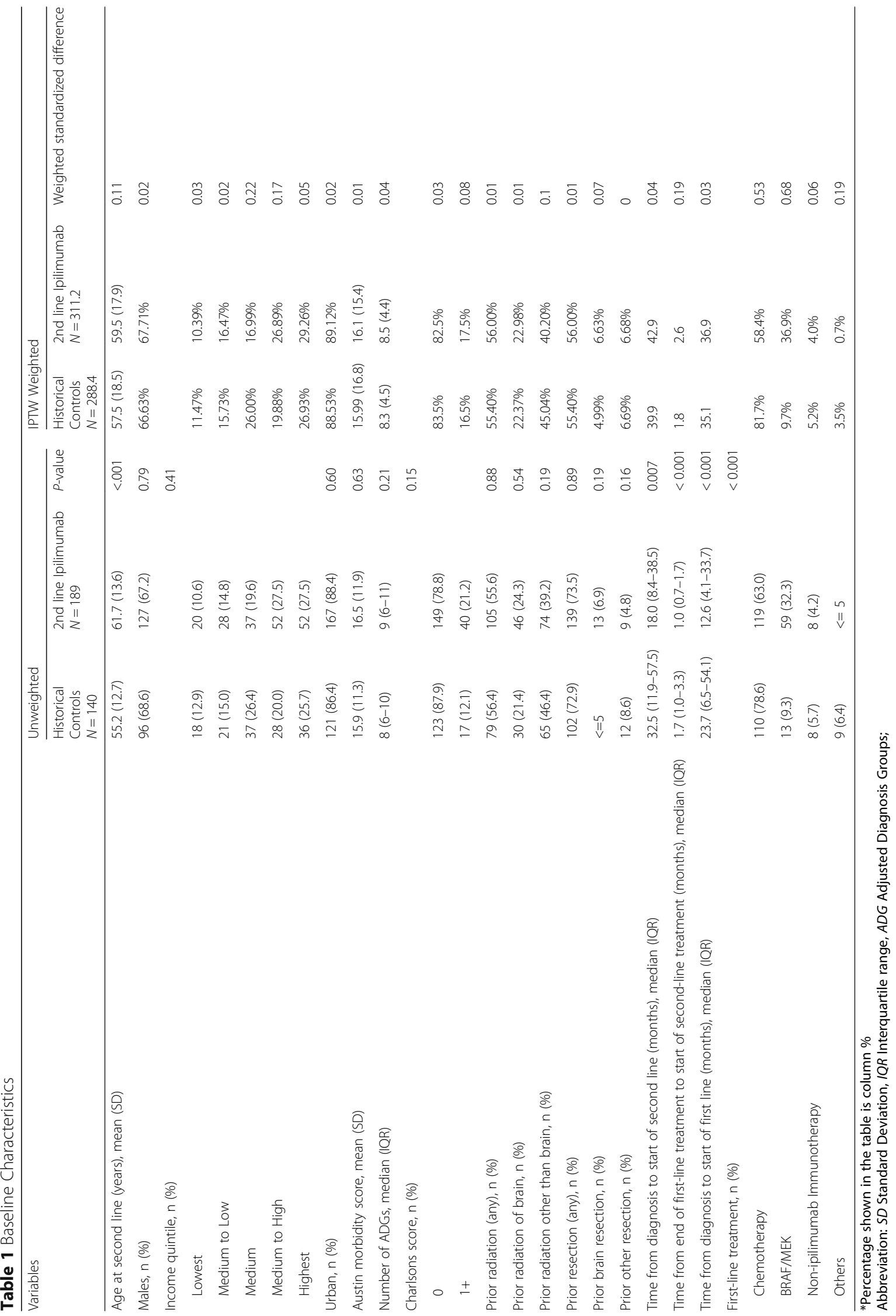


Table 2 Survival Outcomes

\begin{tabular}{lll}
\hline & Historical Controls & $\begin{array}{c}\text { 2nd line Ipilimumab } \\
N=189\end{array}$ \\
\hline Median follow-up months, (95\% Cl) & $30.4(27.9-37.3)$ & $71.2(70.3-116.5)$ \\
1-year survival rates, (95\% Cl) & & $34.3 \%(27-41 \%)$ \\
$\quad$ Unadjusted & $17.1 \%(11-23 \%)$ & $35.6 \%(27-43 \%)$ \\
IPTW-adjusted & $17.1 \%(11-23 \%)$ & $20.6 \%(15-27 \%)$ \\
2-year survival rates, (95\% Cl) & & $21.1 \%(14-28 \%)$ \\
Unadjusted & $7.1 \%(2.9-11 \%)$ & \\
IPTW-adjusted & $7.1 \%(2.9-11 \%)$ & $15.2 \%(9.6-21 \%)$ \\
3-year survival rates, (95\% Cl) & & $14.3 \%(8.0-21 \%)$ \\
$\quad$ Unadjusted & $4.7 \%(1.2-8.2 \%)$ & $4.7 \%(1.2-8.2 \%)$ \\
IPTW-adjusted & & \\
\hline
\end{tabular}

CI: 5.3-8.7 months) compared to historical controls (4.9 months; 95\% CI: 4.3-6.0 months). OS was significantly improved for patients receiving second-line ipilimumab after IPTW adjustment ( $p$-value $<0.0001)$ (Fig. 2b).

Survival was higher at 1-year, 2-year, and 3-year for the ipilimumab group compared to controls in both the unadjusted and IPTW-adjusted analysis (Table 2). Moreover, we observed sustained benefit in the 3 year OS in the ipilimumab group (IPTW-adjusted: 14.3\%; 95\% CI: $8-21 \%)$. Second-line ipilimumab is significantly associated with improved OS in the unadjusted and IPTW weighted Cox proportional hazard model (Unadjusted $\mathrm{HR}=0.65$; 95\% CI: 0.52-0.82; IPTW HR $=0.62$; 95\% CI: 0.52-0.73) (Table 3).

\section{Sensitivity analysis}

Sensitivity analyses were conducted to explore the effect of third line treatment on the association between second-line ipilimumab and OS (Table 3 Models C-F). Despite increasingly conservative assumptions, the estimated ipilimumab treatment effect remained largely unchanged. An interaction term between second-line ipilimumab and third-line treatment, added to models $\mathrm{C}$ and $\mathrm{D}$, was not significant ( $\mathrm{p}_{\text {interaction }}=0.085$ and 0.12 , respectively). Among those patients who received second line ipilimumab and started on third line treatment, the choice of treatment was not significantly associated with survival (immunotherapy vs. other treatment: $\mathrm{HR}=0.75$; 95\% CI: 0.46-1.22 p = 0.25).

\section{Discussion}

In our real-world population-based study, we observed improved survival associated with second-line ipilimumab compared with second-line chemotherapy or targeted therapy (median OS 6.9 vs 4.9 months; Unadjusted $\mathrm{HR}=0.65$; Adjusted $\mathrm{HR}=0.62$ ). The estimated treatment effect was robust under different methods to adjust for the effect of subsequent treatment (HR range $=0.63-$ 0.67). Moreover, despite more older patients receiving second-line ipilimumab (mean age: 61.7 years) compared to chemotherapy or targeted treatment (mean age: 55.2 years) in the real-world, the observed relative treatment effect was still consistent with those reported in the pivotal trial $(\mathrm{HR}=0.66 ; 95 \% \mathrm{CI}$ : 0.51-0.87) [2]. Additionally, we observed approximately half of the second-line ipilimumab patients completed the planned four doses. This was slightly lower than the $64.2 \%$ of patients who received all doses in the trial [2].

In this study, the hazard ratio for overall survival (Unadjusted $\mathrm{HR}=0.65$; Adjusted $\mathrm{HR}=0.62)$ was similar to the pivotal trial $(\mathrm{HR}=0.66)$. The survival curve also plateaued around two to three years, similar to the pivotal trial. In contrast, the relative estimate of survival benefit, median OS, was shorter in the real-world compared to the pivotal trial for both the ipilimumab patients and the control patients. In particular, the real-world median OS was 2.9 months shorter (Real-world: 7.2 months; RCT: 10.1 months) in the ipilimumab group and 1.5 months shorter in the control group (Real-world: 4.9 months; RCT: 6.4 months), compared to clinical trial outcomes. As such, the magnitude of benefit for OS observed in the trial was approximately 3.7 months whereas the realworld effectiveness was 2.3 months. In our real-world setting, less ipilimumab patients were alive at 2-years (Real-world: 21.1\%; RCT: 25\%) and 3-years (Real-world: 14.3\%; RCT: 25\%) [4]. Similarly, less control patients were alive at 2-years (Real-world: $7.1 \%$; RCT: $17 \%$ ) and 3-years (Real-world: 4.7\%; RCT: 10\%) [4]. This difference may be attributable older patients receiving second-line ipilimumab in the real-world as compared to the trial (mean age: 61.7 vs 56.8 years). Moreover, 10\% of treated and $14 \%$ of controls in the trial had central nervous system (CNS) metastases at baseline and received previous treatments for it. In our real-world study, a little over $20 \%$ of patients in each group had radiation to the brain 
A: Unadjusted Kaplan-Meier Survival Curves

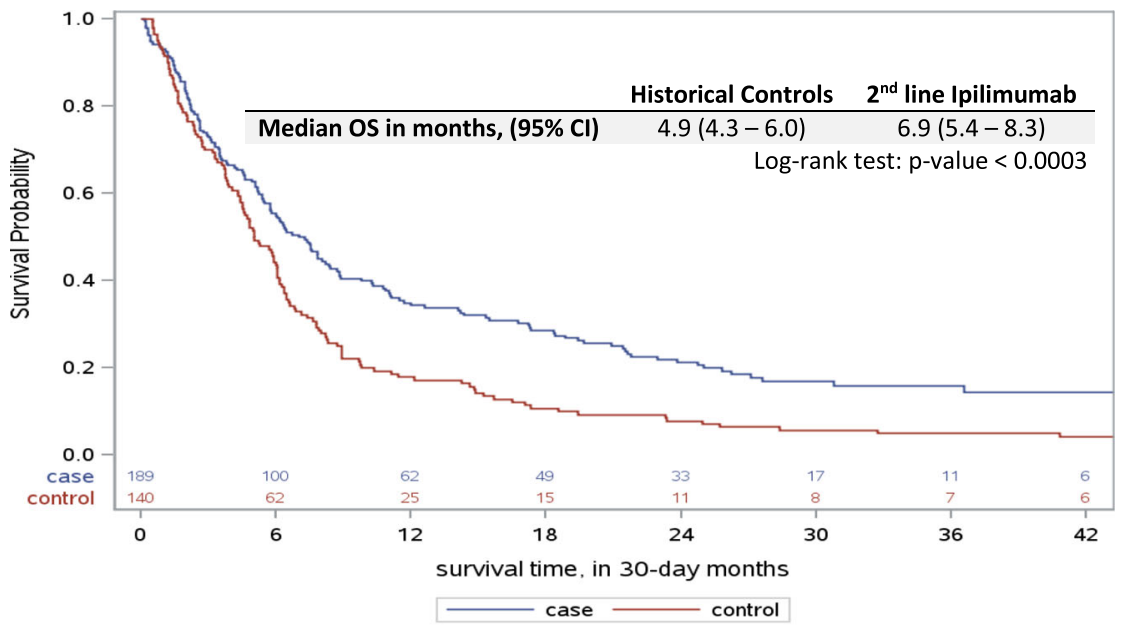

B: IPTW Weighted Kaplan Meier Survival Curves

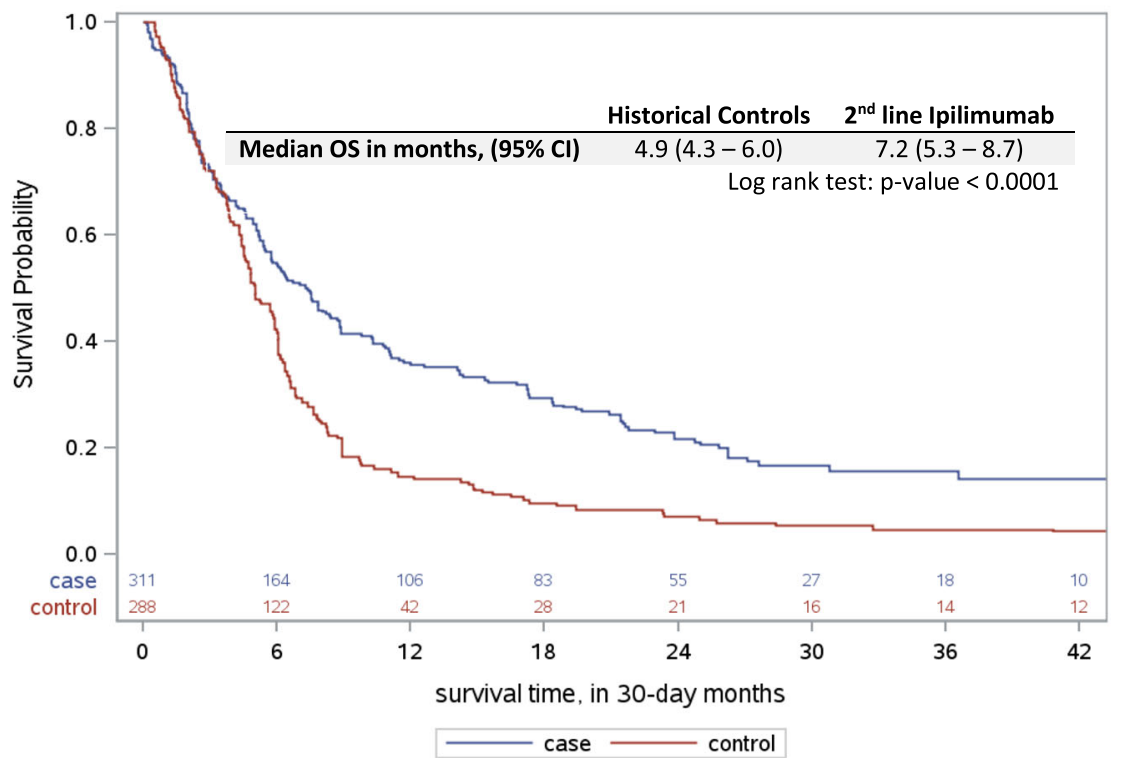

Fig. 2 Overall survival with 2nd line ipilimumab and historical controls (a) Unadjusted (b) IPTW weighted

prior to index systemic treatment, which may have affected absolute survival estimates.

In the population-based study conducted by Polkowska et al., which also compared second-line ipilimumab to second-line chemotherapy, the OS and HR of second-line ipilimumab was similar to our study and the trial. While the median OS were similar between the controls in both studies, patients receiving second-line ipilimumab in our study (median OS: 7.2; 95\% CI: 5.3-8.7) had a slightly higher median OS than those patients in Poland (median OS: 5.9 ; 95\% CI: 5.6-8.4), though the confidence intervals were overlapping between the two studies. Additionally, difference in median OS may also be affected by the difference in access to treatments. The authors mentioned that immunotherapies are only available for second and subsequent lines of treatment. While first-line immunotherapies were not publicly funded in Ontario at the time of our cohorts, around 5\% of patients in each group had received non-ipilimumab immunotherapies from clinical trials or private payers. Furthermore, the third-line treatment availability may also differ between the two studies. In our study, controls were more likely to receive third-line ipilimumab while treated were more likely to receive third-line nivolumab or pembrolizumab. Despite the differences, both observed median OS for second-line ipilimumab was within the range observed in some of the single-arm studies, which ranged between 6.4 to 8.8 months [10-14]. 
Table 3 Hazard Ratio for Overall Survival \& Sensitivity Analysis (2nd line ipilimumab vs historical controls)

\begin{tabular}{|c|c|c|c|}
\hline & & Hazard Ratio $(95 \% \mathrm{Cl})$ & $P$-value \\
\hline \multirow[t]{2}{*}{ Primary Analyses } & Model A: Unadjusted Model & $0.65(0.52-0.82)$ & 0.0003 \\
\hline & Model B: IPTW Weighted Model & $0.62(0.52-0.73)$ & $<0.0001$ \\
\hline \multirow[t]{10}{*}{ Sensitivity Analyses } & Model C: IPTW Weighted Model adjusting for 3rd line & $0.64(0.53-0.76)$ & $<0.0001$ \\
\hline & $\begin{array}{l}\text { Model D: IPTW Weighted Model adjusting for 3rd line } \\
\text { checkpoint inhibitor treatment }\end{array}$ & & \\
\hline & 2nd line Ipilimumab & $0.63(0.53-0.75)$ & $<0.0001$ \\
\hline & Historical Controls & Ref & \\
\hline & Model E: Censoring patients at start of 3rd line & & $<0.0001$ \\
\hline & 2nd line Ipilimumab & $0.60(0.48-0.73)$ & \\
\hline & Historical Controls & Ref & \\
\hline & Model F: Excluding patients who started 3rd line treatment & & 0.0001 \\
\hline & 2nd line Ipilimumab ( $n=122)$ & $0.67(0.55-0.81)$ & \\
\hline & Historical Controls $(n=102)$ & Ref & \\
\hline
\end{tabular}

Our study has a number of notable strengths. We had an extensive collection of linked data, providing detailed information on patient characteristics and treatments for an entire population. Thus, we were able to adjust for many more potential confounders using propensity score methods in contrast to other population-based studies. These variables include rurality, socioeconomic status, previous resection, comorbidity status, and time from diagnosis to initiating second-line treatment. Additionally, we also used different sensitivity analyses to explore the effect of subsequent third-line treatment given that real-world data are non-randomized and that subsequent therapies can be a potential source of confounding. The survival benefit of second-line ipilimumab persisted after these adjustments.

Our study has several limitations. First, inherent to observational studies, our estimates may have been affected by residual confounding from unbalanced or unmeasured variables such as performance status, lactate dehydrogenase, and body mass index. Our analysis did not adjust for performance status, though we were able to control for differences in comorbidity, which could affect functional status. Additionally, given this is a prefunding vs post-funding comparison, it is unlikely that the distribution of performance status of the population would change significantly. Data on lactate dehydrogenase (LDH) was also not available, though notably in the pivotal trial by Hodi et al., there was no significant difference in ipilimumab treatment effect based on LDH [2], and it is unlikely that there would be imbalances given the pre/post design with nearly all patients receiving ipilimumab after funding. Second, in contrast to the randomized trial, the comparator in our study consists of historical controls, since once second-line ipilimumab became available, a very small number of patients received chemotherapy or targeted therapy. While historical controls avoid the small sample size of controls or confounding by indication after ipilimumab funding, historical comparators might be confounded by secular trends such as changes in clinical practice (e.g. radiation or resection practices for metastases, availability of other systemic therapies (see Additional file 1 for public funding timeline) that might bias in favor of ipilimumab. Lastly, after second-line ipilimumab was funded, some patients may have truncated their first-line treatment to access ipilimumab for second-line treatment as soon as possible, as there would have had been no option for first-line immunotherapy. This funding change may have resulted in differences in the extent of disease at the start of second line treatment between treatment groups. Drysdale et al. shown that $40 \%$ of patients who received first-line ipilimumab for MM had received prior chemotherapies for less than 60 days during a similar study period in Ontario [15]. Time between diagnosis to second-line treatment was adjusted for via the propensity score method to address potential bias, though residual imbalance remained in our study.

In addition to validating the efficacy observed in clinical trials, our study also informs policy decisions in Ontario. While ipilimumab was the first immunotherapy to enter the treatment landscape, other immunotherapies, PD1 inhibitors pembrolizumab and nivolumab, have become available in both first-line and second-line setting in Ontario, along with nivolumab in combination with ipilumumab [16-19]. Despite the availabilities of these therapies, the effectiveness of second-line ipilimumab is still of importance for patients who may not be able to tolerate aggressive immunotherapy or progress on combination targeted treatments. Potential future area of investigation can examine the effectiveness of second-line ipilimumab after first-line PD-1 inhibitors and the comparative effectiveness between ipilimumab monotherapy 
and combination therapies. Additional future research relevant for policy decision includes the comparative toxicity of second-line ipilimumab and the effect of immunotherapy on patient symptoms, especially for patients who experience metastases to other body regions at baseline.

\section{Conclusion}

Overall, our findings illustrate the real-world comparative effectiveness of second-line ipilimumab, which was associated with improved survival in patients with MM compared to historical controls, and with HRs similar to those observed in the pivotal trial and other populationbased studies. While the relative treatment effect was similar to the pivotal trial, the median overall survival absolute treatment effect observed among real-world patients treated with ipilimumab was smaller and similar to that observed in the other population-based studies.

\section{Supplementary information}

Supplementary information accompanies this paper at https://doi.org/10. 1186/s12885-020-06798-1.

Additional file 1: Appendix: Figure 1: Funding timeline of metastatic melanoma treatments in Ontario. Table 1: Summary of administrative databases.

\section{Abbreviations}

MM: Metastatic melanoma; HR: Hazard ratio; OS: Overall survival; ALR: Activity Level Reporting; ACG: Adjusted Clinical Group; IPTW: Inverse Probability Treatment Weighting; SD: Standard deviation; IQR: Interquartile Range; $\mathrm{Cl}$ : Confidence Interval

\section{Acknowledgements}

None applicable.

\section{Authors' contributions}

WFD, JB, WI, IM, TP, NM, CE, SG, TH, and KC contributed to the conception and design of the study. WFD, JB, RC, WI, IM, TP, TH, and KC contributed to the analysis of the data. WFD, JB, RC, WI, IM, TP, CC, TH, and KC contributed to the interpretation of the data. WFD, JB, RC, IM, TP, NM, CC, SG, TH, and KC contributed to the writing and editing of the manuscript. TH and KC contributed equally as senior authors. All authors have read and approved to publish this manuscript.

\section{Funding}

This study was funded by the CATALYST grant for Health Services and Economic Research in Cancer - (Grant \# FRN151290) provided by Canadian Institutes of Health Research to Dr. Kelvin Chan.

This study was supported by ICES, which is funded by an annual grant from the Ontario Ministry of Health and Long-Term Care (MOHLTC). This study also received funding from CIHR. Parts of this material are based on data and information compiled and provided by the Canadian Institute for Health Information, Cancer Care Ontario, and the MOHLTC. The analyses, conclusions, opinions and statements expressed herein are solely those of the authors and do not reflect those of the funding or data sources; no endorsement is intended or should be inferred. We thank IMS Brogan Inc. for use of their Drug Information Database for drug identification.

T.P. Hanna holds a research chair provided by the Ontario Institute for Cancer Research through funding provided by the Government of Ontario (\#IA-035).

This project was supported by the Canadian Centre for Applied Research in Cancer Control (ARCC). ARCC receives core funding from the Canadian Cancer Society (Grant \#2015-703549).

\section{Availability of data and materials}

The data that support the findings of this study are available from ICES, but restrictions apply to the availability of these data, which were used under license for the current study, and so are not publicly available. Data are however available from the authors upon reasonable request and with permission of ICES.

Ethics approval and consent to participate

The study has received ethics approval from the Research Ethics Board of Sunnybrook Health Sciences Centre, Ontario, Canada.

\section{Consent for publication}

Not applicable.

\section{Competing interests}

The authors declare that they have no competing interests.

\section{Author details}

${ }^{1}$ Cancer Care Ontario, Toronto, ON, Canada. ${ }^{2}$ Canadian Centre for Applied Research in Cancer Control, Toronto, ON, Canada. ${ }^{3}$ Institute of Clinical Evaluative Sciences, Toronto, ON, Canada. ${ }^{4}$ St Michael's Hospital, Toronto, ON, Canada. ${ }^{5}$ Institute of Health Policy, Management and Evaluation, University of Toronto, Toronto, ON, Canada. ${ }^{6}$ Department of Medicine, University of Toronto, Toronto, ON, Canada. ${ }^{7}$ Sunnybrook Health Sciences Centre, 2075 Bayview Avenue, Toronto, Toronto, Ontario M4N 3M5, Canada. ${ }^{8}$ Division of Cancer Care and Epidemiology, Queen's University Cancer Research Institute, Kingston, Canada. 'Department of Oncology, Queen's University, Kingston, ON, Canada. ${ }^{10}$ Institute of Clinical Evaluative Sciences, Queen's University, Kingston, ON, Canada.

Received: 17 January 2020 Accepted: 27 March 2020

Published online: 15 April 2020

\section{References}

1. Lakdawalla DN, Shafrin J, Hou N, et al. Predicting real-world effectiveness of Cancer therapies using overall survival and progression-free survival from clinical trials: empirical evidence for the ASCO value framework. Value Heal. 2017. https://doi.org/10.1016/j.jval.2017.04.003.

2. Hodi FS, O'Day SJ, McDermott DF, et al. Improved survival with ipilimumab in patients with metastatic melanoma. N Engl J Med. 2010. https://doi.org/ 10.1056/NEJMoa1003466.

3. Korn EL, Liu PY, Lee SJ, et al. Meta-analysis of phase II cooperative group trials in metastatic stage IV melanoma to determine progression-free and overall survival benchmarks for future phase II trials. J Clin Oncol. 2008. https://doi.org/10.1200/JCO.2007.12.7837.

4. McDermott D, Haanen J, Chen TT, Lorigan P, O'Day S. Efficacy and safety of ipilimumab in metastatic melanoma patients surviving more than 2 years following treatment in a phase III trial (MDX010-20). Ann Oncol. 2013. https://doi.org/10.1093/annonc/mdt291.

5. Polkowska M, Ekk-Cierniakowski P, Czepielewska E, Wysoczański W, Matusewicz W, Kozłowska-Wojciechowska M. Survival of melanoma patients treated with novel drugs: retrospective analysis of real-world data. J Cancer Res Clin Oncol. 2017. https://doi.org/10.1007/s00432-017-2453-z.

6. Statistics Canada. Annual demographic estimates : Canada, Provinces and Territories:; 2018.

7. Austin PC, Van Walraven C. The mortality risk score and the ADG score: two points-based scoring systems for the Johns Hopkins aggregated diagnosis groups to predict mortality in a general adult population cohort in Ontario, Canada. Med Care. 2011. https://doi.org/10.1097/MLR.0b013e318229360e.

8. Sundararajan V, Henderson T, Perry C, Muggivan A, Quan H, Ghali WA. New ICD-10 version of the Charlson comorbidity index predicted in-hospital mortality. J Clin Epidemiol. 2004. https://doi.org/10.1016/j.jclinepi.2004.03. 012.

9. Austin PC. An introduction to propensity score methods for reducing the effects of confounding in observational studies. Multivariate Behav Res. 2011. https://doi.org/10.1080/00273171.2011.568786.

10. Jochems A, Leeneman B, Franken MG, et al. Real-world use, safety, and survival of ipilimumab in metastatic cutaneous melanoma in the Netherlands. Anti-Cancer Drugs. 2018. https://doi.org/10.1097/CAD. 0000000000000629. 
11. Russi A, Damuzzo V, Chiumente M, et al. Ipilimumab in real-world clinical practice: efficacy and safety data from a multicenter observational study. J Chemother. 2017. https://doi.org/10.1080/1120009X.2017.1311444.

12. Middleton MR, Dalle S, Claveau J, et al. Real-world treatment practice in patients with advanced melanoma in the era before ipilimumab: results from the IMAGE study. Cancer Med. 2016. https://doi.org/10.1002/cam4.717.

13. Khoja L, Atenafu EG, Ye Q, et al. Real-world efficacy, toxicity and clinical management of ipilimumab treatment in metastatic melanoma. Oncol Lett. 2016. https://doi.org/10.3892/ol.2015.4069.

14. Krajsová I, Arenberger P, Lakomý R, et al. Long-term survival with ipilimumab: experience from a national expanded access program for patients with melanoma. Anticancer Res. 2015;35(11):6303-10.

15. Drysdale E, Peng Y, Nguyen P, Baetz T, Hanna TP. A population-based study of the treatment effect of first-line ipilimumab for metastatic or unresectable melanoma. Melanoma Res. 2019. https://doi.org/10.1097/CMR. 0000000000000582.

16. Ribas A, Puzanov I, Dummer R, et al. Pembrolizumab versus investigatorchoice chemotherapy for ipilimumab-refractory melanoma (KEYNOTE-002): a randomised, controlled, phase 2 trial. Lancet Oncol. 2015. https://doi.org/10. 1016/S1470-2045(15)00083-2.

17. Robert C, Schachter J, Long GV, et al. Pembrolizumab versus Ipilimumab in Advanced Melanoma. N Engl J Med. 2015. https://doi.org/10.1056/ NEJMoa1503093.

18. Robert C, Long GV, Brady B, et al. Nivolumab in previously untreated melanoma without BRAF mutation. N Engl J Med. 2015. https://doi.org/10. 1056/NEJMoa1412082

19. Hodi FS, Chesney J, Pavlick AC, et al. Combined nivolumab and ipilimumab versus ipilimumab alone in patients with advanced melanoma: 2 -year overall survival outcomes in a multicentre, randomised, controlled, phase 2 trial. Lancet Oncol. 2016. https://doi.org/10.1016/S1470-2045(16)30366-7.

\section{Publisher's Note}

Springer Nature remains neutral with regard to jurisdictional claims in published maps and institutional affiliations.

Ready to submit your research? Choose BMC and benefit from:

- fast, convenient online submission

- thorough peer review by experienced researchers in your field

- rapid publication on acceptance

- support for research data, including large and complex data types

- gold Open Access which fosters wider collaboration and increased citations

- maximum visibility for your research: over $100 \mathrm{M}$ website views per year

At $\mathrm{BMC}$, research is always in progress.

Learn more biomedcentral.com/submissions 